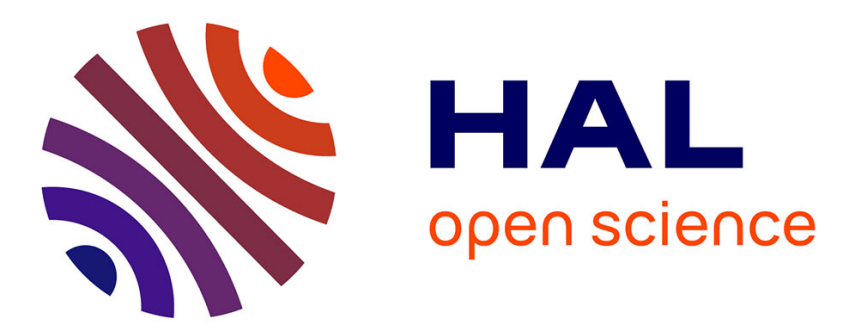

\title{
Environmental systems modeling and diagnosis using a multiple model approach
}

Komi Gasso, Gilles Mourot, José Ragot

\section{To cite this version:}

Komi Gasso, Gilles Mourot, José Ragot. Environmental systems modeling and diagnosis using a multiple model approach. 5th International FLINS Conference, Sep 2002, Gent, Belgium. pp.387-394. hal-00510893

\section{HAL Id: hal-00510893 \\ https://hal.science/hal-00510893}

Submitted on 6 Apr 2014

HAL is a multi-disciplinary open access archive for the deposit and dissemination of scientific research documents, whether they are published or not. The documents may come from teaching and research institutions in France or abroad, or from public or private research centers.
L'archive ouverte pluridisciplinaire HAL, est destinée au dépôt et à la diffusion de documents scientifiques de niveau recherche, publiés ou non, émanant des établissements d'enseignement et de recherche français ou étrangers, des laboratoires publics ou privés. 


\title{
ENVIRONMENTAL SYSTEMS MODELLING AND DIAGNOSIS USING A MULTIPLE MODEL APPROACH
}

\author{
Komi Gasso, Gilles Mourot and José Ragot
}

\begin{abstract}
Centre de Recherche en Automatique de Nancy - CNRS UMR 7039, Ecole Nationale Supérieure de Géologie. 2, Avenue de la Forêt de Haye - 54516 Vandoeuvre-lès-Nancy, France

Email: \{ggasso,gmourot,jragot\}@ensem.inpl-nancy.fr
\end{abstract}

\begin{abstract}
The paper aims to describe the problem of modelling non-linear dynamic systems using a multiple model representation, also known in fuzzy set literature as Takagi-Sugeno model. The basic principle of this approach is to represent the system as an interpolation of locally valid models. Proposed identification algorithms concern the parameter estimation and the structure determination (local model orders, number of local models). Three applications of this approach to environmental system management are reported: modelling of a wastewater treatment plant, ozone concentration prediction is an urban area and finally modelling of rainfall-runoff relationship for sensor fault diagnosis in urban sewage network.
\end{abstract}

Keywords: non-linear dynamic system; time series prediction; system identification; multiple model; fuzzy model; environmental systems; ozone concentration; sensor fault diagnosis; wastewater treatment plant, rainfall-runoff relationship.

\section{Introduction}

Developing a mathematical model of a process can be motivated by many reasons: process control or diagnosis, its behaviour prediction, estimation of unmeasurable variables or even more simply to improve our understanding about the system behaviour. A possible way to handle this problem is the "white box" modelling that is solely based on the knowledge of the physical principles taking place in the system. However, excepted for relatively simple systems, the construction of a physical model is quite difficult because the involved phenomena are very complex and mathematically difficult to describe, especially for environmental systems (lots of complex non linearities, long and variable delays, timevarying systems, no exact physical models available, possibility of a high level of noise, ...). Moreover some important variables are seldom available and some physical parameters may be unknown. Therefore, a model constructed from the data extracted on the process is preferable; this kind of model is also known as "black box" model. Different types of model structures have been proposed for non-linear systems identification among which NARMAX structures (Chen and Billings, 1989) and Wiener - Hammerstein type models (Haber and Unberhauen, 1990). These classical methods attempt to find a global model capable to represent the system over its full operating range. The obtained model is consequently complex. An elegant way to avoid this difficulty is the multiple model approach (Johansen and Foss, 1993) that represents the system as an interpolation of simple (usually linear) local models. Each local model describes the behaviour of the system in a limited part of the operating space. The local validity of the submodels is specified by corresponding weighting functions which provide a smooth transition between them. Notice that in this sense, the wellknown Takagi-Sugeno fuzzy model (Takagi and Sugeno, 1985) is a particular multiple model defined as a set of fuzzy rules "If premise then linear local behaviour". In the sequel, the mathematical formulation of the multiple model is presented as well as the tasks related to the 
identification of such a model. The application to the modelling of environmental systems is reported in the last section.

\section{Mathematical formulation of the multiple model}

Consider a non-linear Multi-Input/Single Output dynamic system. The multiple model approach represents the system as a local model network described by the equation:

$$
y(k)=\sum_{i=1}^{M} \omega_{i}(z(k), \beta) f_{i}\left(\varphi(k), \theta_{i}\right)
$$

$f_{i}$ indicates a local model which depends on a regression vector $\varphi(k)$ and on a local parameter vector $\theta_{i}$. The weighting function $\omega_{i}$ associated to each model $f_{i}$ acts as a local validity measure of this submodel according to the current operating regime of the system. The vector $\beta$ parameterises these weighting functions. They are defined over the operating space $Z$ spanned by the vector $z(k)$ and their number and position determine the "fuzzy" partition of Z. The vector of feature variables $z(k)$ can include lagged inputs and output of the system or any auxiliary variable allowing the non linearities of the process to be taken into account. The weighting functions are defined such as they verify the following constraints:

$$
\sum_{i=1}^{M} \omega_{i}(z(k), \beta)=1 \quad \forall k, \quad \omega_{i}(z(k), \beta) \geq 0 \quad \forall k, \forall i
$$

Numerous weighting functions can be proposed; commonly they are chosen as normalized gaussian functions or can also be constructed from sigmoidal functions.

The local models could have different structures but here, for the sake of simplicity, we assume that they share the same inputs with the same orders. The local models are expressed by the relation:

$$
y_{i}(k)=f_{i}\left(\varphi(k), \theta_{i}\right)=\varphi^{T}(k) \theta_{i} \quad i=1, \mathrm{~L}, M
$$

where the regression vector is defined as:

$$
\begin{aligned}
\varphi^{T}(k)= & {\left[-y(k-1) \mathrm{K}-y\left(k-n_{y}\right) u_{1}(k-d) \mathrm{K}\right.} \\
& \left.u_{1}\left(k-d-n_{u}\right) \ldots u_{m}(k-d) \mathrm{K} u_{m}\left(k-d-n_{u}\right)\right]
\end{aligned}
$$

It includes the delayed inputs $u_{r}(r=1, \ldots, m)$ and output $y$ of the model. $n_{y}$ and $n_{u}$ are the orders and $d$ the delay of the local models; $m$ is the number of inputs.

The next sections discuss the problems involved by the identification of a multiple model.

\section{Identification issues of a multiple model}

Identify a multiple model involves mainly two tasks: the parameter estimation and the structure identification. The principles of the solutions provided to these problems are exposed below. 


\subsection{Parameter estimation}

Let $\theta^{T}=\left[\begin{array}{llll}\theta^{T}{ }_{1} & \theta^{T} & \ldots & \theta^{T}\end{array}\right]$, the vector of local model parameters. The parameters $\theta$ of the local models and those $\beta$ of the weighting function are estimated in order to minimise the following criterion:

$$
J=\frac{1}{2} \sum_{k=k_{0}}^{N}\left(y(k)-y_{m}(k)\right)^{2} \quad k_{0}=\max \left(n_{y}, d+n_{u}\right)+1
$$

with $y_{m}$ the measured output of the system and $N$ the number of training data. As the criterion $J$ is non-linear with the vectors $\theta$ and $\beta$, their estimation is achieved trough a non-linear optimisation technique like the Levenberg-Marquardt (LM) method. For practical reasons related to the great number of parameters that can be involved in a multiple model, a twolevel algorithm (Boukhris, et al., 1999) is used: it consists in computing $\beta$ by a LM algorithm for $\theta$ fixed and estimating $\theta$ by another LM algorithm for the parameters $\beta$ previously determined. The procedure is repeated until convergence.

However, by substituting the measured output $y_{m}$ to the model output $y$ in the regression vector (see eq. 4), it can be shown that the criterion $J$ becomes quadratic with respect to $\theta$ which can be easily computed by the least squares method. Based on this remark, another two-level algorithm has been developed (Mourot et al., 1999), (Gasso et al., 1999): the vector $\theta$ is estimated by least squares for fixed value of $\beta$; $\theta$ being known, the vector $\beta$ is optimised using a non-linear optimisation method. This is iterated until convergence.

\subsection{Structure optimization}

It concerns the characterisation of the operating space $Z$, the partition of $Z$ in operating areas (that determines the number of local models) and the identification of the orders and delays of these submodels.

In the multiple model representation, simultaneous determination of the structure and the number of local models is difficult because a trade-off must be achieved between the number and the complexity of the local models. Indeed, few complex submodels are needed to approximate adequately a system and conversely. Therefore, this interdependency forces to proceed sequentially. An elegant way to solve this problem is to choose, a priori an initial and common structure for all the local models and to identify their appropriate number and position in the operating space (Tanaka, et al., 1995). The priori choice of the local model structure can be suggested by using the modified Lipschitz quotient (Boukhris et al., 1999).

The number of local models in the structure is related to the partitioning adopted for the operating space. Grid partitioning is very simple to realise. However, the number of submodels increases exponentially (curse of dimensionality) with the number of feature variables and/or the number of modality per feature variable. Owing to the fact that the feature space is rarely uniformly covered by training data and due to its combinatorial aspect, the grid partition may produce:

- near empty or empty subspaces. The local models associated to these subspaces are less important because their contribution to the explanation of the behaviour of the system is negligible,

- redundant submodels i.e. neighbouring local models that can provide the same description of the system but are arbitrary separated by the grid partition. 
Therefore, in order to identify a parsimonious multiple model, the number of local models can be reduced by deleting the irrelevant submodels and merging the compatible neighbouring local models. We have proposed a technique described in (Gasso et al., 2001). The procedure can generate operating regimes with arbitrary boundaries, unlike the axis orthogonal subspaces in the grid partition. This reduces the final number of local models.

The drawback of the grid partition can be avoided by using more local partitioning techniques. Tree partitioning such as a k-d tree allows areas to be defined along a corresponding decision tree (Johansen et Foss, 1995), (Nelles, 1997), (Gasso et al., 1999). Consequently, the number of local models can be reduced. Indeed, this method doesn't produce near empty or empty subspaces but may produce redundant local models.

\section{Application to environmental system management}

In the following, the works described above are illustrated on three different environmental systems. These three system deal with the following application fields: process modeling for control and optimization (modeling of a water treatment plant), time series prediction (forecasting of ozone concentration in an urban area) and sensor fault diagnosis (sensor failure detection in an urban sewer network).

\subsection{Modeling of a water treatment plant (Ragot et al., 2001)}

The system studied is a pilot wastewater treatment plant whose objective is the treatment of municipal wastewater of the Greater Nancy Urban Authority. From the viewpoint of the manager of this plant, the objective is compliance with the standards for municipal effluent quality (the chemical characteristics of the purified water at the plant, even if the quality of the raw water supplied to the process varies over time. This adaptation must be as rapid as possible for reasons of safety and economy. Its implementation makes it necessary for operating personnel to have greater expertise, and for the personnel to be provided with adequate information and explanations about how the process works. In an optimization stage, the improvement of the quality of these effluents is also envisaged.

In general, to quantify the impurities present in effluents, the concept used is that of Chemical Oxygen Demand (COD) an image of which can be supplied at the plant outlet by a measure of UV absorption (globally, this variable reflects the concentration of organic matter present in the treated effluent). In the plant in question, the COD can be modified by using sludge activated by bacterial culture. The principle of the control of the process lies in the controlled development of a bacteria floc in the aeration tanks supplied by the effluent to be treated; a flow of oxygen enables the proliferation of the microorganisms, which biologically purify the effluent. Some of the sludge from settling is recycled to the aeration tank inlet to promote reseeding with bacteria. The variables, which characterize the process, are set out in table 1 .

\begin{tabular}{|l|l|}
\hline \multicolumn{2}{|c|}{ Input variables } \\
\hline A & Turbidity $\mathrm{TU}_{\mathrm{e}}$ \\
\hline B & Absorption $\mathrm{UV}_{\mathrm{e}}$ \\
\hline $\mathrm{C}$ & Visible absorption \\
\hline D & Temperature $\mathrm{T}_{\mathrm{e}}$ \\
\hline E & Tank 1 oxygen concentration \\
\hline $\mathrm{H}$ & Tank 1 pH \\
\hline $\mathrm{I}$ & Tank 1 eH \\
\hline J & Desaerator sludge concentration \\
\hline
\end{tabular}

\begin{tabular}{|l|l|}
\hline \multicolumn{2}{|c|}{ Command variables } \\
\hline F & Tank 2 air flow \\
\hline G & Tank 3 air flow \\
\hline
\end{tabular}

\begin{tabular}{|l|l|}
\hline \multicolumn{2}{|c|}{ Output variables } \\
\hline $\mathrm{L}$ & Turbidity \\
\hline $\mathrm{M}$ & Absorption $^{\mathrm{UV}_{\mathrm{S}}}$ \\
\hline $\mathrm{N}$ & Visible absorption \\
\hline
\end{tabular}

Table 1: process variables 
Various measurement campaigns have been conducted on this during which significant variations in the different variables were recorded.

In the case in hand, the explanatory variables are turbidity $T U_{e}$, absorption $U V_{e}$ and temperature $T_{e}$ of the inlet effluent, the use of supplementary variables providing no significant contribution as regards the behavior of the variable $U V_{s}$. The weight functions, for the three inputs, have been chosen as trapezoidal form and the final model involves four local models. The temporal evolutions of the prediction supplied by the model and of the corresponding measurement show the good performances of the model. This comparison has also been performed on two other measurement campaigns and confirms the first results obtained.

As the modeling of the other outlet variables (turbidity and visible absorption) have been performed in a similar way, particular interest will be focused on the $\mathrm{UV}_{\mathrm{s}}$ variable of the effluent at the plant outlet.

\subsection{Modeling of ozone concentration (Mourot et al., 1999, Gasso et al., 1999)}

The problem under investigation is the modelling of ozone in collaboration with air quality monitoring networks in Lorraine (eastern France) in order to forecast daily maximum ozone concentration. Ozone is a pollutant in the lower troposphere. It has detrimental effects on human health and on environment when its concentration reaches excessive values. In situations of high ozone level, appropriate decisions must be taken by authorities to inform the public and possibly to control the phenomenon. Therefore, the development of models is necessary to estimate ozone level in order to anticipate the decisions.

Ozone is a secondary pollutant produced by complex photochemical reactions between nitrogen oxides (mainly $\mathrm{NO}$ and $\mathrm{NO}_{2}$ ) and Volatile Organic Compounds emitted into the atmosphere. These reactions depend highly on the precursors emissions level and on the vertical and horizontal movements of the atmosphere that are linked to the meteorological conditions. By way of their interaction, these physical and chemical elements constitute a dynamic non-linear, multivariable and time-varying process. The theoretical models of ozone comprise the description of the physico-chemical mechanisms of ozone production and destruction and combine a large number of equations. Unfortunately, there is incomplete knowledge of the overall mechanisms. Furthermore, these models are computationally costly and they need measurements which are rarely available in air quality monitoring network. Therefore, they are hardly use in practice and black-box modelling of the ozone concentration has to be performed.

In this study, the concentrations of several pollutants, as well as meteorological variables, are measured (Table 2).

\begin{tabular}{|lr|}
\hline \multicolumn{2}{|c|}{ Input Variables } \\
\hline Temperature & ${ }^{\circ} \mathrm{C}$ \\
\hline Solar radiation & $\mathrm{W} / \mathrm{m}^{2}$ \\
\hline Nitrogen oxide NO & $\mu \mathrm{g} / \mathrm{m}^{3}$ \\
\hline Nitrogen dioxide $\mathrm{NO}_{2}$ & $\mu \mathrm{g} / \mathrm{m}^{3}$ \\
\hline Relative humidity & $\%$ \\
\hline Pressure & $\mathrm{hPa}$ \\
\hline Wind Direction & degree \\
\hline Wind speed & $\mathrm{m} / \mathrm{s}$ \\
\hline
\end{tabular}

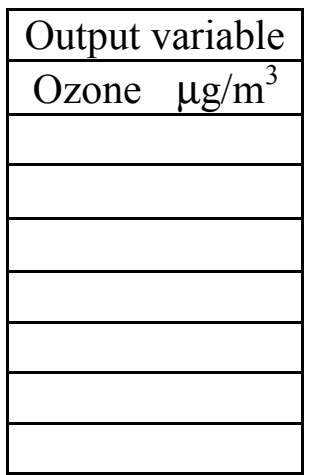

Table 2. Characteristic variables of the phenomenon 
The best model structure uses the following variables: temperature $\left(u_{1}\right)$, solar radiation $\left(u_{2}\right)$, $\mathrm{NO}_{2}\left(u_{3}\right)$ and windspeed $\left(u_{4}\right)$. The weight functions have been chosen as trapezoidal form. The model retained to characterize the evolution of ozone is described by six local models.

Results concerning the modelling of an air pollution phenomenon illustrate the outcome that could be expected in using the proposed approach; for relatively short observation periods (a few days) the comparison between the measured ozone concentration and its prediction by the multiple model is excellent. Moreover the selected variables take account of the main chemical and meteorological factors that play a part in the mechanisms of ground-level ozone formation. The validation of the structure obtained for much longer observation periods (in the order of 12 months) and the testing of some predictive qualities remain to be carried out, and will form the basis of the continuation of this study. It could also be postulated that the processing of pollution data from several geographical locations would again improve the model quality.

\subsection{Rainfall-runoff modeling for sensor fault diagnosis (Boukhris et al.,2001)}

Twenty years ago, to prevent urban flooding, the metropolitan authorities of Nancy - France, Communauté Urbaine du Grand Nancy (CUGN), opted for detention basin construction and sewer rehabilitation to improve storm-water management. Since completion of this step, the $C U G N$ is now interested in the quality of water introduced into the receiving environment, namely the Meurthe river. The constraints of flood prevention and pollution control now require more reliable measurements provided by different sensors.

Failure detection and isolation has received considerable attention and many applications in high technology industries, and safety-critical processes like nuclear power plants, chemical plants and transportation systems have been proposed. However, few applications have been dedicated to environmental processes, in particular to urban sewerage networks. This may be due to the specificity of these systems, namely complex non-linearity, time-varying, unavailable physical models and the nature of measurements available (laboratory analyses, variable sampling time...).

Traditionally, failure detection and isolation has been achieved through the use of simple (or direct) redundancy. Simple redundancy consists of increasing the number of sensors in a triplex or quadruplex configuration which measures the same physical variable, and redundant measurements are compared for consistency. This approach can be simple and in some cases, reasonably straightforward to apply and is thus widely used. The major problem encountered with hardware redundancy is the extra cost and the overall reliability is not necessarily improved. Furthermore, the proposed sensor failure detection and isolation method has to be sufficiently flexible to be extended to actuator (pumps, gates...) failure detection and isolation.

Actually, the model-based approach is an alternative that is generally based on consistency checking between an observed process behavior, provided by sensors, and an expected behavior provided by a model of the process or parts thereof. It is based on the use of analytical rather than hardware redundancy. Indeed, the inherent analytical redundancies contained in the relationships between the measured system outputs helps to deduce virtual (or software) sensors that act as supplementary sensors.

In this particular application, the problem of sensor fault diagnosis requires the availability of a rainfall - runoff relationship in order to apply analytical redundancy-based diagnostic procedures. The problem of relating rainfall to runoff, for operational purposes, is often tackled in one of two ways. The system's approach aims to represent the overall process behavior through mathematically simple relationships, whereas, in the conceptual modelling 
approach, the behavior of the process is modelled in a simplified way reflecting known physical laws. In the conceptual approach, several parts of the model are easily recognized as representing various stages in the hydrological cycle. However, the physical complexity and non-linearity of the phenomenon and the usual unavailability of distributed data result in a broad simplification of such models. In the system's approach, linear time-invariant models are usually used but are often inefficient except perhaps for very small watersheds. But, even in this case, non-linear models give a better performance than linear ones. This has prompted the investigation of an alternative method which still falls within the domain of system approach.

The proposed multiple modelling approach has been successfully tested on a watershed located in an urban area of Nancy, in eastern France, using actual rainfall and runoff data taken from the sewerage control centre database. The global model is described by nine multiple models. This model has then been used to implement a sensor fault diagnostic procedure (one sensor measuring the rainfall intensity and two sensors measuring the flowrates at the watershed's output). Furthermore, the proposed sensor failure detection and isolation method has to be extended to actuator (pumps, water gates...) failure detection and isolation.

\section{Conclusion}

Developing a mathematical model of a process can be motivated by many reasons: process control or diagnosis, its behavior prediction, estimation of unmeasurable variables or even more simply to improve our understanding about the system behavior. However, few applications have been dedicated to environmental processes. This may be due to the specificity of these systems, namely lots of complex non-linearity, time-varying, unavailable physical models and the nature of measurements available (laboratory analyses, variable sampling time...). An elegant way to overcome these difficulties is the multiple model approach that represents the system as a smooth interpolation of simple (usually linear) local models. Each local model describes the behavior of the system in a limited part of the operating space.

Although this type of model is well adapted to the representation of a system with non-linear behavior, its use does however raise real problems in the identification of its structure and parameters. The problems are mainly due to the combinatorial explosion of the complexity of the model as a function of the number of inputs, as well as the difficulty of identifying the operating regime and the local models, which are mutually related. Methods for the identification of a multiple model (structure and parameters) have proposed.

These methods are illustrated on three different environmental systems for the following application fields: process modeling for control and optimization (modeling of a water treatment plant), time series prediction (forecasting of ozone concentration in an urban area) and sensor fault diagnosis (sensor failure detection in an urban sewer network).

\section{References}

Boukhris, A., G. Mourot and J. Ragot (1999). Non-linear dynamic system identification: a multiple-model approach. Int. Journal of control, vol. 72, N7/8, pp. 591-604.

Boukhris, A., S. Giuliani, G. Mourot (2001). Rainfall-runoff multi-modelling for sensor fault diagnosis. Control Engineering Practice, vol. 9, pp. 659-671. 
Chen S. and Billings S. A. (1989) "Representation of non-linear systems: the NARMAX model". Int. Journal of Control, vol. 49, N³, pp. 1013-1032.

Gasso K., Mourot G. and Ragot J. (1999) "Ozone concentration modelling using a multiple model approach". Proc. of EUFIT'99. Aachen, Germany.

Gasso, K., G. Mourot and J. Ragot (2001). Structure identification in multiple model representation: elimination and merging of local models. $C D C^{\prime} 2001$, Orlando, Florida, 2001.

Johansen T. A. and Foss A. B. (1993) "Constructing Narmax using Armax". Int. Journal of Control, vol. 58, N5, pp 1125-1153.

Johansen, T. A. and A. B. Foss (1995). Identification of non-linear system structure and parameters using regime decomposition. Automatica, vol. 31, N², pp. 321-326.

Takagi, T. and Sugeno, M. (1985). Fuzzy identification of systems and its application to modelling and control. IEEE Trans. on Systems Man and Cybernetics, 15, 116-132.

Nelles, O., A. Fink, R., Babuška and Setnes M. (1999). Comparison of two construction algorithms for Takagi-Sugeno fuzzy models. Proc. of Eufit'99, Aachen, Germany, 1999.

Ragot J., Grapin G., Chatellier P., Colin F. (2001). Modelling of water treatment plant. A multi-model representation. Environmetrics, vol. 12, pp. 599-611.

Tanaka, K., M. Sano and H. Watanabe (1995). Modelling and control of carbon monoxide concentration using a neuro-fuzzy technique. IEEE Trans. on Fuzzy Systems, vol. 3, N³, pp. 271-279. 